\title{
Instrumentos desenvolvidos para o gerenciamento e cuidado de idosos em instituições de longa permanência: uma revisão sistemática
}

\author{
Instruments developed for the management and care \\ of the elderly in long-stay care institutions: a systematic review
}

Paulo Adão de Medeiros ${ }^{1}$

Artur Rodrigues Fortunato ${ }^{1}$

Adriana Aparecida da Fonseca Viscardi ${ }^{1}$

Fabiana Flores Sperandio ${ }^{1}$

Giovana Zarpellon Mazo ${ }^{1}$

${ }^{1}$ Centro de Ciências

da Saúde e do Esporte,

Universidade do Estado de

Santa Catarina. R. Pascoal

Simone 358, Coqueiros.

88080-350 Florianópolis

SC Brasil.

paulofisiosm@yahoo.com.br
Abstract The demand for long-stay care institutions for the elderly (ILPIs) is increasing and it is relevant for public health systems to discuss the process of assessing the health status of its residents. The study aimed to identify measurement tools built specifically for the management and care of elderly residents in long-stay care facilities. A systematic review was conducted following PRISMA recommendations in Medline and CINAHL databases since their creation to May 2013 using the Medical Subject Headings terms suitable for the search. A total of 1858 articles were located, of which 30 were selected and 28 instruments were identified in the studies. The United States was the country that created most instruments geared to this population and the Minimum Data Set/ Resident Assessment Instrument (MDS/RAI) was the one most used in the studies of this review. The variables most frequently evaluated by the instruments were depression, cognition and functional capacity. The reformulation of public policies that ensure a standardized assessment system for residents in ILPIs in Brazil is urgently needed and the challenge is to ensure that the instruments developed are disseminated and effectively implemented in the daily tasks of the professionals working in these institutions.

Key words Elderly, Institutionalization, Measurements, Methods and theories, Questionnaires, Review
Resumo Como a demanda por instituições de longa permanência para idosos (ILPIs) está aumentando, torna-se relevante para os sistemas de saúde pública discutir o processo de avaliação das condições de saúde dos seus residentes. O presente estudo objetivou identificar instrumentos de medida construídos especificamente para o gerenciamento e o cuidado de residentes idosos, em instituições de longa permanência. Realizouse uma revisão sistemática segundo as recomendações PRISMA, nos bancos de dados Medline e CINAHL, desde a sua criação até maio de 2013, utilizando termos da Medical Subject Headings adequados para a busca. Foram encontrados 1858 artigos e selecionados 30 , sendo identificados 28 instrumentos nesses estudos. Os EUA foram o país que mais construiu instrumentos voltados a essa população e o Minimum Data Set/Resident Assessment Instrument (MDS/RAI) foi o mais utilizado nos estudos da presente revisão. As variáveis mais frequentemente avaliadas pelos instrumentos foram a depressão, a cognição e a capacidade funcional. Torna-se urgente a reformulação de políticas públicas que garantam um sistema de avaliação padronizado dos residentes de ILPIs no Brasil, sendo um desafio fazer com que os instrumentos desenvolvidos consigam se difundir e se efetivar no cotidiano dos profissionais dessas instituições.

Palavras-chave Idoso, Institucionalização, $\mathrm{Me}$ didas, Métodos e teorias, Questionários, Revisão 


\section{Introdução}

Acompanhando o aumento da população idosa, a demanda por cuidados de longa duração tende a aumentar em todo o mundo, gerando problemas de saúde pública ${ }^{1-3}$. Com isso, surgem as Instituições de Longa Permanência para Idosos (ILPIs) que são organizações complexas, constituídas, majoritariamente, de subsistemas internos, em que a equipe multiprofissional deve se ocupar dos processos de gerenciamento e cuidado da saúde global do idoso ${ }^{1,4}$.

Nessa perspectiva, torna-se função das ILPIs promover a manutenção e a melhoria da qualidade de vida desses idosos, com um monitoramento constante que permita auxiliar no melhor manejo dos residentes ${ }^{5}$. Contudo, Felician et al. ${ }^{6}$ declararam a inexistência de instrumentos que avaliem as ILPIs, sob a ótica do cuidado. Para suprir tal deficiência, desenvolveram um estudo preliminar com o propósito de construir um instrumento que identificasse as necessidades reais de uma instituição e de seus residentes. No entanto, existem fortes evidências de que essa temática seja atual, relevante e careça de ser constantemente estudada.

Diante deste fato, entende-se que embora a saúde do idoso tenha sido anteriormente pesquisada, as discrepâncias e as inadequações na coleta de informações no ato de admissão destes, como residentes, contribuem para uma análise enviesada das condições de saúde, o que pode dificultar a adoção de estratégias eficazes no atendimento dessa população. A falta de homogeneidade de informações dificulta a construção de protocolos que ofereceriam subsídios concretos na tomada de decisões clínicas a curto e longo prazo 7 .

Instrumentos de medida são partes integrantes da prática clínica, da avaliação em saúde e de pesquisas, sendo úteis e capazes de apresentar resultados robustos quando desenvolvidos e utilizados cientificamente ${ }^{8}$. Assim, os métodos e as técnicas de coleta de informações devem garantir indicadores seguros e confiáveis ${ }^{9}$, em que a tomada de decisão vai depender do desenho da pesquisa e do quanto tais instrumentos são adequados e precisos na avaliação das variáveis de interesse. Portanto, torna-se necessário um mapeamento sistemático e elaborado dos instrumentos de avaliação disponíveis ${ }^{10,11}$.

Os idosos residentes em Instituições de Longa Permanência necessitam de constante monitoramento das condições de saúde, o que é previsto na legislação brasileira ${ }^{12,13}$. Sendo assim, entendese que esses idosos possuem características pecu- liares que precisam ser consideradas na sua avaliação. Nesse sentido, instrumentos construídos especificamente para esse público são relevantes para mensurar adequadamente o seu perfil, bem como a realidade vivenciada no contexto institucional. No entanto, existe uma lacuna de discussão sobre o processo de avaliação dos residentes nas ILPIs brasileiras e não se encontrou revisões que buscassem traçar um panorama geral sobre os instrumentos de avaliação desenvolvidos especificamente para essa população.

Assim, pressupõe-se que investigações desta natureza possam oferecer o conhecimento dos distintos tipos de ferramentas utilizados em situações de rotina clínica, quer seja no processo de admissão tanto quanto na análise dos efeitos de uma possível intervenção sobre os aspectos físico -emocionais, organizacionais ou multidimensionais. Tais resultados e interpretações oferecerão informações a respeito da produção científica sobre o tema, com orientações claras acerca das propriedades destes instrumentos de medida tão heterogêneos, podendo auxiliar profissionais e pesquisadores no seu processo de trabalho.

Diante deste contexto e dos argumentos supracitados, o presente estudo objetivou identificar, por meio de revisão sistemática, instrumentos de medida construídos especificamente para o gerenciamento e o cuidado de residentes idosos, em instituições de longa permanência.

\section{Método}

Esta revisão sistemática foi realizada de acordo com as orientações Preferred Reporting Items for Systematic Reviews and Meta-Analyses ${ }^{14,15}$.

\section{Estratégias de busca e critérios de seleção}

As bases de dados selecionadas foram $\mathrm{Me}$ dical Literature Analysis and Retrieval System Online - Medline (OVID WEB; de 1966 a 27 de maio de 2013) e Cumulative Index to Nursing and Allied Health Literature - CINAHL (de 1982 a 27 de maio de 2013). Assim, foram examinadas desde o primeiro registro até maio de 2013, quando ocorreu a extração dos dados. Optou-se pela Medline, pois é uma das bases de dados mais utilizadas pelas ciências da saúde e contém uma extensa literatura, abrigando no seu banco também referências do PubMed. E a CINAHL, uma das bases mais utilizadas pela enfermagem, uma das profissões que mais trabalha e pesquisa idosos institucionalizados. 
As estratégias de pesquisa estão descritas no Quadro 1 e originaram-se a partir do Medical Subject Headings (MeSH). Optou-se por designar três blocos de análises, quais sejam: Idoso, Institucionalização e Estudos diagnósticos, sendo este último filtro baseado nas recomendações para estudos diagnósticos fornecidos pelo Scottish Intercollegiate Guidelines Network ${ }^{16}$.

Os resultados da pesquisa foram exportados para um sistema de gestão de referências (Zotero ${ }^{\circledR}$ versão 3.0) que permitiu a identificação e a exclusão de registros duplicados.

O processo de triagem dos estudos ocorreu em duas etapas:
Etapa 1: Foram definidos os seguintes critérios de inclusão: (1) estudos sobre construção ou validação de instrumentos de medida; (2) amostra composta apenas por idosos institucionalizados; (3) idioma inglês, espanhol ou português. Foram excluídos estudos com dados secundários (revisões), resumos, artigos indisponíveis na íntegra e, ainda, cujo instrumento fosse de avaliação da ILPI ou para previsão do processo de institucionalização do idoso residente na comunidade.

Seguindo esses critérios, primeiro foram analisados os títulos dos estudos selecionados. Posteriormente, foram avaliados os resumos dos

Quadro 1. Estratégias de pesquisa nas bases de dados escolhidas: MEDLINE (OVID) e CINAHL.

\begin{tabular}{|c|c|}
\hline MEDLINE (OVID WEB) & CINAHL \\
\hline $\begin{array}{l}1 \text { Aged/ } \\
2 \text { Age.tw. } \\
3 \text { Elderly.tw. } \\
4 \text { Older.tw. } \\
5 \text { (Old }^{*} \text { adj2 People).tw. } \\
\left.6 \text { (Old }{ }^{*} \text { adj2 Adult }{ }^{*}\right) . t w . \\
7 \text { Aging/ } \\
8 \text { or } / 1-7\end{array}$ & 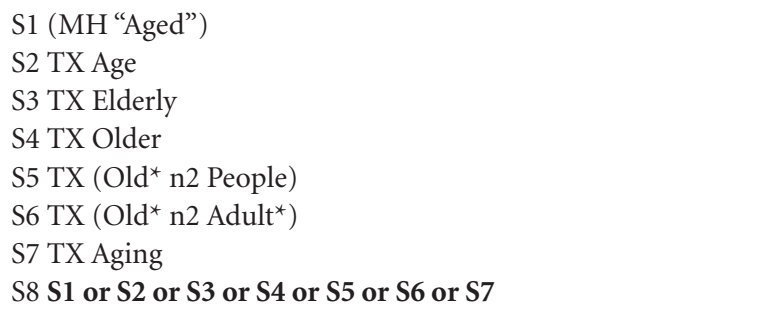 \\
\hline $\begin{array}{l}9 \text { Institutionalization/ } \\
10 \text { Health services for the aged/ } \\
11 \text { Almshouses/ } \\
12 \text { Homes for the aged/ } \\
13 \text { Housing for the elderly/ } \\
14 \text { Long-term care/ } \\
15 \text { (Institutionalized elderly).tw. } \\
16 \text { (Geriatric Long-term care).tw. } \\
17 \text { Charities.tw. } \\
18 \text { (Sheltered housing).tw. } \\
19 \text { (Housing for older).tw. } \\
20 \text { (Senior housing).tw. } \\
21 \text { or/9-20 }\end{array}$ & $\begin{array}{l}\text { S9 (MH "Institutionalization") } \\
\text { S10 (MH "Health services for the aged") } \\
\text { S11 TX (Almshouses) } \\
\text { S12 TX (Homes for the aged) } \\
\text { S13 (MH "Housing for the elderly") } \\
\text { S14 (MH "Long-term care") } \\
\text { S15 TX (Institutionalized elderly) } \\
\text { S16 TX (Geriatric Long-term care) } \\
\text { S17 (MH "Charities") } \\
\text { S18 TX (Sheltered housing) } \\
\text { S19 TX (Housing for Older) } \\
\text { S20 TX (Senior housing) } \\
\text { S21 S9 or S10 or S11 or S12 or S13 or S14 or S15 or S16 or S17 } \\
\text { or S18 or S19 or S20 }\end{array}$ \\
\hline $\begin{array}{l}22 \text { exp “Sensitivity and Specificity”/ } \\
23 \text { sensitivity.tw. } \\
24 \text { specificity.tw. } \\
25 \text { ((pre-test or pretest) adj probability).tw. } \\
26 \text { post-test probability.tw. } \\
27 \text { predictive value\$.tw. } \\
28 \text { likelihood ratio\$.tw. } \\
29 \text { or/ } 22-28\end{array}$ & $\begin{array}{l}\text { S22 (MH “Sensitivity and Specificity") } \\
\text { S23 TX sensitivity } \\
\text { S24 TX specificity } \\
\text { S25 TX (pre-test or pretest) n2 probability } \\
\text { S26 TX post-test probability } \\
\text { S27 TX predictive value\$ } \\
\text { S28 TX likelihood ratio } \$ \\
\text { S29 S22 or S23 or S24 or S25 or S26 or S27 or S28 }\end{array}$ \\
\hline 308 and 21 and 29 & $S 30 S 8$ and $S 21$ and S29 \\
\hline
\end{tabular}

Fonte: composição dos autores. 
estudos remanescentes e, por fim, foi considerada a importância dos artigos na íntegra. Todo o processo de leitura e avaliação dos artigos foi realizado por dois avaliadores de forma independente e as divergências entre os revisores foram resolvidas por discussão abrangente até obtenção de consenso.

Etapa 2: Após a seleção, foi acrescentado mais um critério de exclusão na tentativa de focar somente em estudos voltados ao desenvolvimento de instrumentos para avaliar o idoso institucionalizado. Sendo assim, foram excluídos os estudos de validação de instrumentos que foram desenvolvidos em outros contextos e que seriam, secundariamente, validados para aplicabilidade nas ILPI $(\mathrm{n}=30)$. Ainda, nessa etapa, cada artigo foi submetido a uma análise detalhada com o intuito de identificar outros instrumentos de medida, como: escalas, testes, inventários, questionários, checklists ou índices que foram desenvolvidos ou adaptados especificamente para a realidade dos residentes em ILPIs. Para tanto, foi realizada a checagem das referências de todos os instrumentos mencionados no método.

As ILPIs representam instituições bastante heterogêneas no mundo e não existe uma definição internacional em comum para identificá-las. Por esse motivo, analisou-se os principais termos utilizados em publicações científicas da área. Adicionalmente, em todas as etapas de leitura e na seleção dos artigos, houve a preocupação de se fazer uma análise criteriosa com o intuito de identificar os estudos referentes a Instituições de Longa Permanência para Idosos.

\section{Extração dos dados}

A partir dos estudos selecionados, foram elaborados formulários padronizados para o fichamento das principais informações de interesse que posteriormente foram checadas pelos revisores para a apresentação dos dados no estudo.

\section{Resultados}

Essa busca resultou em 1858 títulos (685 no Medline e 1173 no CINAHL) - destes, 98 eram duplicados e foram retirados, ficando 1760 para a leitura dos títulos. Assim, restaram 287 para a leitura do resumo e, finalmente, 161 para a análise na íntegra. Ao final da etapa 1 e 2 , foram incluídos 60 e 30 artigos, respectivamente. No total, foram identificados 28 instrumentos para a análise, conforme ilustra a Figura 1.

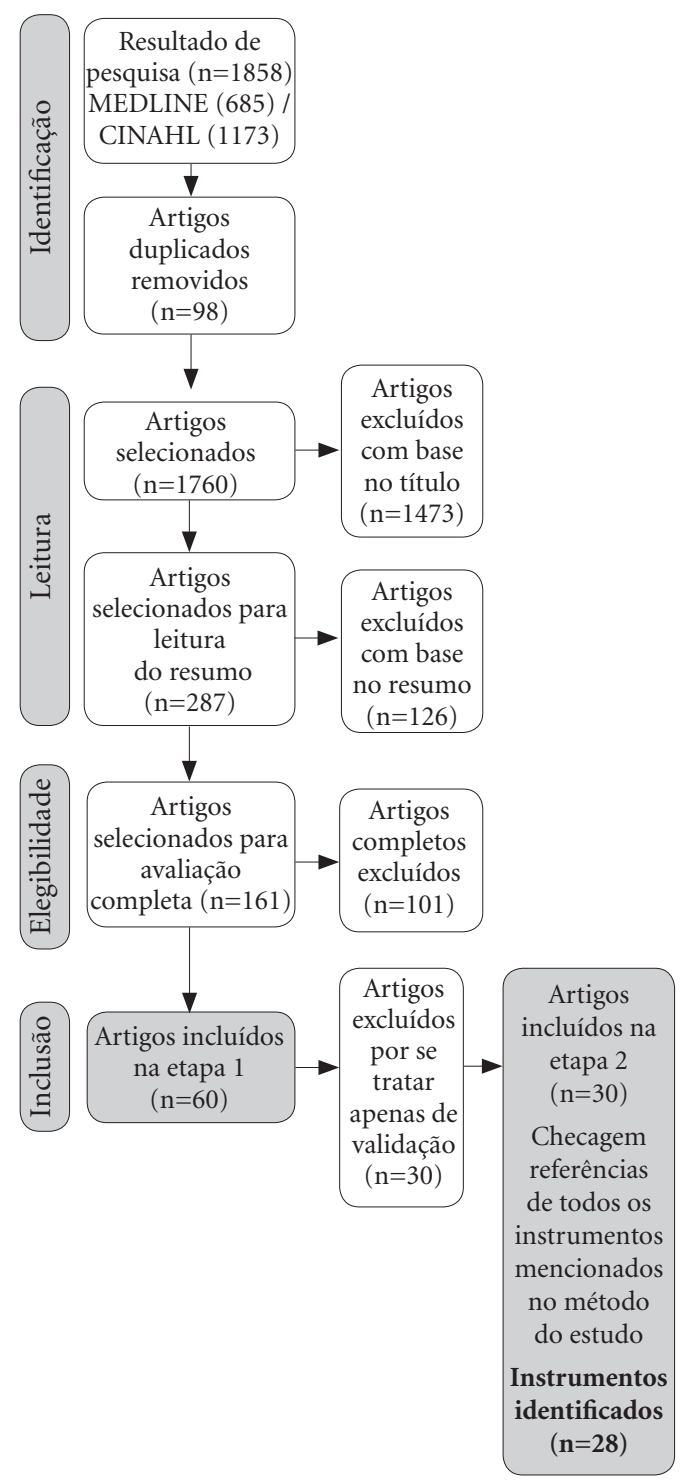

Figura 1. Fluxograma do processo de seleção dos artigos.

Fonte: composição dos autores.

No Quadro 2 estão representados os 30 artigos selecionados na revisão, com as referências, número $\left(\mathrm{n}^{\circ}\right)$ e nome dos instrumentos identificados em cada estudo.

Após a identificação dos instrumentos de avaliação desenvolvidos para o contexto das ILPIs, procurou-se verificar suas principais características, os quais foram separados por aspectos semelhantes e estão representados no Quadro 3. 
Quadro 2. Referências, número e nome dos instrumentos específicos para avaliação dos residentes em ILPIs encontrados em cada estudo $(n=30)$.

\begin{tabular}{|c|c|c|c|}
\hline Referências & No e Nome dos instrumentos & Referências & No e Nome dos instrumentos \\
\hline Frisoni et al. ${ }^{17}$ & 1-Índice Nutricional - NUTRIX & Resnick e Jung ${ }^{34}$ & $\begin{array}{l}\text { 1-Maryland Assisted Living } \\
\text { Functional Assessment (MALFA) }\end{array}$ \\
\hline \multirow[t]{2}{*}{ Hartmaier et al..$^{18}$} & \multirow{2}{*}{$\begin{array}{l}\text { 1-MDS Cognition Scale (MDS- } \\
\text { COGS) } \\
\text { 2-MDS-RAI } \\
\text { 3-Cognitive Performance Scale } \\
\text { (CPS) }\end{array}$} & Jongenelis et al. ${ }^{35}$ & $\begin{array}{l}\text { 1-Geriatric Depression Scale } \\
\text { (GDS 8) } \\
\text { 2-GDS-12R }\end{array}$ \\
\hline & & \multirow{2}{*}{ Paquay et al..$^{36}$} & \multirow[b]{2}{*}{$\begin{array}{l}\text { 1-MDS-RAI } \\
\text { 2-The Belgian ADL-Evaluation } \\
\text { Scale } \\
\text { 3-Cognitive Performance Scale } \\
\text { (CPS) }\end{array}$} \\
\hline Björkgren et al. ${ }^{20}$ & 1-Resource Utilization & & \\
\hline Björkgren et al. ${ }^{20}$ & $\begin{array}{l}\text { 1-Resource Utilization } \\
\text { Groups, Version III (RUG-III) }\end{array}$ & Auret et al. ${ }^{37}$ & 1-Modified Residents Verbal Brief \\
\hline \multirow[t]{2}{*}{ Burrows et al. ${ }^{21}$} & \multirow{2}{*}{$\begin{array}{l}\text { 1-Minimum Data Set Depression } \\
\text { Rating Scale } \\
\text { 2-MDS-RAI }\end{array}$} & & Pain Inventory (M-RVBPI) \\
\hline & & Chodosh et al. ${ }^{38}$ & $\begin{array}{l}\text { 1-Brief Interview of Mental Status } \\
\text { (BIMS) }\end{array}$ \\
\hline \multirow[t]{2}{*}{$\begin{array}{l}\text { Gruber-Baldini } \\
\text { et al. }{ }^{22}\end{array}$} & \multirow{2}{*}{$\begin{array}{l}\text { 1-Cognitive Performance Scale } \\
\text { (CPS) } \\
\text { 2-MDS Cognition Scale (MDS- } \\
\text { COGS) } \\
\text { 3-MDS-RAI }\end{array}$} & & $\begin{array}{l}\text { 2-MDS-RAI } \\
\text { 3-Cognitive Performance Scale } \\
\text { (CPS) }\end{array}$ \\
\hline & & Delbaere et al. ${ }^{39}$ & $\begin{array}{l}\text { 1-Modelos de rastreio } \\
\text { desenvolvidos para o estudo }\end{array}$ \\
\hline Sutcliffe et al. ${ }^{23}$ & 1-GDS-12R & \multirow{3}{*}{ Gerritsen et al. ${ }^{40}$} & \multirow{3}{*}{$\begin{array}{l}\text { 1-Index for Social Engagement } \\
\text { (ISE) } \\
\text { 2-Cognitive Performance Scale } \\
\text { (CPS) } \\
\text { 3-MDS-RAI }\end{array}$} \\
\hline Teresi et al..$^{24}$ & $\begin{array}{l}\text { 1-Research Mattis Dementia } \\
\text { Rating Scale (RMDRS) }\end{array}$ & & \\
\hline \multirow[t]{3}{*}{ Dijkstra et al. ${ }^{25}$} & \multirow{3}{*}{$\begin{array}{l}\text { 1-Dutch Care Dependency Scale } \\
\text { (CDS) } \\
\text { 2-The Nursing Minimal } \\
\text { Data Set for the Netherlands } \\
\text { (NMDSN) }\end{array}$} & & \\
\hline & & Thorsell et al. ${ }^{41}$ & $\begin{array}{l}\text { 1-Time in Care Needs (TIC-n) } \\
\text { instrument (19-item version) }\end{array}$ \\
\hline & & Worden et al. ${ }^{42}$ & 1-MDS-RAI \\
\hline McCurren $^{26}$ & 1-MDS & \multirow{4}{*}{ Huijbregts et al. ${ }^{43}$} & \multirow{4}{*}{$\begin{array}{l}\text { 1-Continuing Care Activity } \\
\text { Measure (CCAM) } \\
\text { 2-MDS-ADL } \\
\text { 3-MDS Cognitive Performance } \\
\text { Scale } \\
\text { 4-MDS-CHESS Scale } \\
\text { 5-MDS-RAI } \\
\text { 6-The RUG-III resident } \\
\text { classification system }\end{array}$} \\
\hline Anderson et al. ${ }^{27}$ & $\begin{array}{l}\text { 1-Minimum Data Set Depression } \\
\text { Rating } \\
\text { 2-Scale (MDSDRS) } \\
\text { 3-MDS-RAI }\end{array}$ & & \\
\hline Oborne et al..$^{28}$ & $\begin{array}{l}\text { 1-Indicadores de qualidade de } \\
\text { prescrição }\end{array}$ & & \\
\hline \multirow[t]{2}{*}{ Algase et al. ${ }^{29}$} & \multirow{2}{*}{$\begin{array}{l}\text { 1-Algase Wandering Scale } \\
\text { (Version 2) (AWSV2) }\end{array}$} & & \\
\hline & & \multirow[t]{4}{*}{ Watson et al. ${ }^{44}$} & \multirow{4}{*}{$\begin{array}{l}\text { 1-Cornell Scale for Depression in } \\
\text { Dementia, modified for use by } \\
\text { long-term care staff (CSDD-M- } \\
\text { LTCS). } \\
\text { 2-Depression Rating Scale } \\
\text { 3-Activities of Daily Living } \\
\text { Subscale of the Minimum Data } \\
\text { Set (MDS-ADL) }\end{array}$} \\
\hline Miller et al. ${ }^{30}$ & $\begin{array}{l}\text { 1-Seating Identification Tool } \\
\text { (SIT) }\end{array}$ & & \\
\hline Stevenson et al. ${ }^{31}$ & 1-MDS-RAI & & \\
\hline \multirow{2}{*}{$\begin{array}{l}\text { Bowman e } \\
\text { Keller }^{32}\end{array}$} & \multirow{2}{*}{$\begin{array}{l}\text { 1-Minimum Data Set (MDS) } 2.0 \\
\text { oral/nutrition status (Section K) }\end{array}$} & & \\
\hline & & Berlowitz et al. ${ }^{45}$ & 1-MDS \\
\hline McGilton et al. ${ }^{33}$ & $\begin{array}{l}\text { 1-Self-Report Relational Care } \\
\text { Scale (RCS) } \\
\text { 2-MDS-RAI }\end{array}$ & Leontjevas et al. ${ }^{46}$ & $\begin{array}{l}\text { 1-Nijmegen Observer-Rated } \\
\text { Depression Scale (NORD) }\end{array}$ \\
\hline
\end{tabular}

Fonte: composição dos autores. 
Quadro 3. Características dos Instrumentos: nome, referência primária do desenvolvimento, país de origem, número de citações dos instrumentos nos 30 artigos identificados nesta revisão, variáveis de avaliação e principais descrições sobre o instrumento desenvolvido para idosos residentes em ILPIs.

\begin{tabular}{|c|c|c|c|c|}
\hline Nome e Referência & País & $\mathrm{N}$ cit & Variáveis & Descrição do instrumento \\
\hline \multicolumn{5}{|c|}{ Aspectos multidimensionais } \\
\hline $\begin{array}{l}\text { Resource Utilization Groups, } \\
\text { Version III (RUGIII) } \\
\text { Fries e Cooney }{ }^{47}\end{array}$ & EUA & 2 & $\begin{array}{l}\text { Custos por } \\
\text { residente }\end{array}$ & $\begin{array}{l}\text { Classifica o residente conforme os custos: 1) reabilitação } \\
\text { especial; 2) serviços intensivos; 3) cuidados especiais; } \\
\text { 4) quadro clínico complexo; 5) cognição prejudicada; } \\
\text { 6) problemas de comportamento; 7) redução da função } \\
\text { física. }\end{array}$ \\
\hline $\begin{array}{l}\text { Minimum Data Set/Resident } \\
\text { Assessment Instrument } \\
\text { (MDS/RAI) }^{* * *} \\
\text { Morris et al. }{ }^{48}\end{array}$ & EUA & 13 & $\begin{array}{l}\text { Saúde, } \\
\text { funcionamento } \\
\text { e bem-estar }\end{array}$ & $\begin{array}{l}\text { Estruturado em } 18 \text { Protocolos de Avaliação dos } \\
\text { Residentes (RAPs). Consiste em: rotinas habituais, } \\
\text { padrões de comunicação, funcionamento físico } \\
\text { e problemas estruturais, padrões de humor e } \\
\text { comportamento, diagnóstico de doenças, estado } \\
\text { nutricional, condições da pele, transtornos e } \\
\text { procedimentos especiais, padrões cognitivos, visão, } \\
\text { continência, continuidade de atividades, condições de } \\
\text { saúde, estado oral e dental e o uso de medicaçôes. }\end{array}$ \\
\hline $\begin{array}{l}\text { Maryland Assisted Living } \\
\text { Functional Assessment } \\
\text { (MALFA) } \\
\text { Resnick e Jung }{ }^{49}\end{array}$ & EUA & 1 & $\begin{array}{l}\text { Necessidades } \\
\text { físicas, } \\
\text { funcionais e } \\
\text { psicossociais }\end{array}$ & $\begin{array}{l}\text { É composto de sete sub-categorias: 1) monitoramento de } \\
\text { doenças; 2) monitoramento de deficiências cognitivas, } \\
\text { abuso de substâncias, e doenças psiquiátricas; } 3 \text { ) execução } \\
\text { de tratamentos para condições físicas; 4) gestão de } \\
\text { medicamentos; 5) assistência com AVDs; 6) gestão de } \\
\text { situações de alto risco; 7) gestão de comportamentos } \\
\text { problemáticos. }\end{array}$ \\
\hline $\begin{array}{l}\text { Time in Care needs - TIC-n } \\
\text { (19-item version) } \\
\text { Thorsell et al. }{ }^{41}\end{array}$ & Suécia & 1 & $\begin{array}{l}\text { Necessidade de } \\
\text { cuidados }\end{array}$ & $\begin{array}{l}\text { Compreende o tempo gasto no cuidado com os } \\
\text { residentes. Possui } 19 \text { itens divididos em: cuidados gerais, } \\
\text { assistência médica e cuidados cognitivos. As pontuações } \\
\text { maiores indicam necessidade de maiores cuidados. }\end{array}$ \\
\hline $\begin{array}{l}\text { Dutch Care Dependency } \\
\text { Scale (CDS) } \\
\text { Dijkstra, Buist e Dassen } \\
\end{array}$ & Holanda & 1 & $\begin{array}{l}\text { Dependência } \\
\text { de cuidados }\end{array}$ & $\begin{array}{l}\text { Possui } 15 \text { itens: alimentação, incontinência, postura } \\
\text { corporal, mobilidade, ciclo dia e noite, capacidade de } \\
\text { vestir-se ou despir-se, temperatura corporal, higiene, } \\
\text { prevenção de perigos, comunicação, contato com } \\
\text { outros, senso de regras e valores, estruturação de } \\
\text { AVDs, atividades fora da instituição, capacidade de } \\
\text { aprendizagem. }\end{array}$ \\
\hline \multicolumn{5}{|c|}{ Aspectos físicos } \\
\hline $\begin{array}{l}\text { Índice Nutricional - } \\
\text { NUTRIX } \\
\text { Frisoni et al. }{ }^{17}\end{array}$ & Itália & 1 & $\begin{array}{l}\text { Predição de } \\
\text { mortalidade }\end{array}$ & $\begin{array}{l}\text { Esse índice é preditivo de mortalidade em idosos de } \\
\text { ILPI, sendo calculado por meio de exames laboratoriais e } \\
\text { variáveis antropométricas. }\end{array}$ \\
\hline $\begin{array}{l}\text { Minimum Data Set (MDS - } \\
\text { Section K) } \\
\text { Canadian Institute for } \\
\text { Health Information }^{* * *}\end{array}$ & EUA & 1 & $\begin{array}{l}\text { Condições } \\
\text { orais e } \\
\text { nutricionais }\end{array}$ & $\begin{array}{l}\text { Questões relacionadas à perda de peso recente, } \\
\text { reclamações quanto ao sabor dos alimentos, à quantidade } \\
\text { de comida que permanece no prato após as refeições, } \\
\text { ao uso de alimentação parenteral ou uso de dieta } \\
\text { terapêutica, além da identificação das úlceras de pressão. }\end{array}$ \\
\hline $\begin{array}{l}\text { Belgian ADL-Evaluation } \\
\text { Scale } \\
\text { Arnaert e Delesie }\end{array}$ & Bélgica & 1 & $\begin{array}{l}\text { Capacidade } \\
\text { Funcional }\end{array}$ & $\begin{array}{l}\text { Avaliação das AVDs em seis domínios originais } \\
\text { (tomar banho vestir-se, transferência, ir ao banheiro, } \\
\text { continência, comer). Foram adicionados } 2 \text { itens (uso da } \\
\text { funcionalidade associada a orientação no tempo e no } \\
\text { espaço da ILPI). }\end{array}$ \\
\hline $\begin{array}{l}\text { Activities of Daily Living } \\
\text { subscale of the Minimum } \\
\text { Data Set (MDS-ADL) } \\
\text { Morris, Fries e Morris }^{* *}\end{array}$ & EUA & 2 & $\begin{array}{l}\text { Capacidade } \\
\text { Funcional }\end{array}$ & $\begin{array}{l}\text { Os itens avaliados são: vestir, higiene pessoal, uso do } \\
\text { banheiro, locomoção na instituição, transferência, } \\
\text { mobilidade na cama, comer (inclui alimentação por } \\
\text { sonda ou nutrição parenteral). }\end{array}$ \\
\hline $\begin{array}{l}\text { MDS-CHESS Scale } \\
\text { Hirdes et al. }{ }^{* 4}\end{array}$ & $\begin{array}{l}\text { EUA e } \\
\text { Canadá }\end{array}$ & 1 & $\begin{array}{l}\text { Predição de } \\
\text { mortalidade }\end{array}$ & $\begin{array}{l}\text { Emprega itens de três áreas do MDS 2.0: alterações na } \\
\text { saúde, com base nas medidas do estado de declínio nos } \\
\text { últimos } 90 \text { dias; doença em fase terminal e sintomas e } \\
\text { sinais de problemas médicos. }\end{array}$ \\
\hline
\end{tabular}


Quadro 3. continuação

\begin{tabular}{|c|c|c|c|c|}
\hline Nome e Referência & País & N cit & Variáveis & Descrição do instrumento \\
\hline $\begin{array}{l}\text { Indicadores de qualidade de } \\
\text { prescrição } \\
\text { Oborne et al. } .^{28}\end{array}$ & Inglaterra & 1 & $\begin{array}{l}\text { Prescrição de } \\
\text { medicamentos }\end{array}$ & $\begin{array}{l}\text { Foram modificados e testados } 13 \text { indicadores já utilizados } \\
\text { em hospitais, relacionados à prescrição baseada em } \\
\text { dados clínicos e evidências científicas, uso adequado de } \\
\text { benzodiazepínicos, esteroides e antitrombóticos, cuidado } \\
\text { com duplicação terapêutica, sensibilidade a drogas, } \\
\text { registro e informações a respeito da administração. }\end{array}$ \\
\hline $\begin{array}{l}\text { Seating Identification Tool } \\
\text { (SIT) } \\
\text { Miller et al. }{ }^{30}\end{array}$ & Canadá & 1 & $\begin{array}{l}\text { Uso/adaptação } \\
\text { à cadeira de } \\
\text { rodas }\end{array}$ & $\begin{array}{l}\text { Avalia cinco áreas sobre características do residente } \\
\text { cadeirante: pressão, comportamentos de desconforto, } \\
\text { mobilidade, posicionamento e estabilidade do paciente. } \\
\text { Os dados são obtidos através da revisão de prontuários } \\
\text { ou autorrelatos. }\end{array}$ \\
\hline $\begin{array}{l}\text { Modified Residents' Verbal } \\
\text { Brief Pain Inventory } \\
\text { (M-RVBPI) } \\
\text { Auret et al. }{ }^{37}\end{array}$ & Austrália & 1 & Dor & $\begin{array}{l}\text { Os itens dizem respeito a: ocorrência, localização e piora } \\
\text { da dor nas últimas } 24 \text { hs, ocorrência e intensidade de } \\
\text { dor ao movimento, o mínimo e máximo de dor sentida, } \\
\text { efeitos sobre a realização de atividades e interação com } \\
\text { outras pessoas nas últimas } 24 \mathrm{hs} \text {. }\end{array}$ \\
\hline $\begin{array}{l}\text { Modelos de rastreio } \\
\text { desenvolvidos para quedas } \\
\text { Delbaere et al. }{ }^{39}\end{array}$ & $\begin{array}{l}\text { Austrália } \\
\text { e Bélgica }\end{array}$ & 1 & $\begin{array}{l}\text { Risco de } \\
\text { quedas }\end{array}$ & $\begin{array}{l}\text { A capacidade de despir-se associada (com falta de } \\
\text { equilíbrio sobre o tapete de espuma, quedas anteriores, } \\
\text { residência em instituição de idosos, incontinência } \\
\text { urinária, polifarmácia) geram modelos preditivos para } \\
\text { quedas. }\end{array}$ \\
\hline $\begin{array}{l}\text { Continuing Care Activity } \\
\text { Measure (CCAM) } \\
\text { Huijbregts et al. }{ }^{43}\end{array}$ & Canadá & 1 & $\begin{array}{l}\text { Função } \\
\text { motora }\end{array}$ & $\begin{array}{l}\text { Contém } 16 \text { itens de avaliação divididos em três } \\
\text { domínios: função motora grossa, mobilidade e membros } \\
\text { superiores. A pontuação varia de } 16 \text { a } 112 \text { pontos e segue } \\
\text { a classificação: Independência, Supervisão, Assistência } \\
\text { (mínima, moderada, máxima ou total). }\end{array}$ \\
\hline \multicolumn{5}{|c|}{ Aspectos psicológicos } \\
\hline $\begin{array}{l}\text { MDS Cognition Scale } \\
\text { (MDS-COGS) }^{* * *} \\
\text { Hartmaier et al. }{ }^{18}\end{array}$ & EUA & 2 & Cognição & $\begin{array}{l}\text { É dividida em padrões cognitivos (memória de curto } \\
\text { e de longo prazo, localização do próprio quarto, saber } \\
\text { que está em uma ILPI, orientação, tomada de decisão); } \\
\text { em padrões de comunicação (se faz entender); e } \\
\text { funcionamento físico (auto desempenho ao vestir-se). }\end{array}$ \\
\hline $\begin{array}{l}\text { Cognitive Performance Scale } \\
\left(\text { CPS }{ }^{* * *}\right. \\
\text { Morris et al. }{ }^{55}\end{array}$ & EUA & 6 & Cognição & $\begin{array}{l}\text { Combina informações principalmente sobre a } \\
\text { capacidade de tomar decisões, de se fazer entender e } \\
\text { perda de memória. Os parâmetros variam (1) em coma; } \\
\text { (2) problema com a memória de curto prazo; (3) as } \\
\text { habilidades cognitivas para a tomada de decisão diária; } \\
\text { (4) ser compreendido por outros; (5) as atividades da } \\
\text { vida diária (AVD) e auto-desempenho em comer. }\end{array}$ \\
\hline $\begin{array}{l}\text { Short DMS-III-R } \\
\text { Isohanni et al. }{ }^{19}\end{array}$ & Finlândia & 1 & $\begin{array}{l}\text { Transtorno } \\
\text { mental }\end{array}$ & $\begin{array}{l}\text { Possui as seguintes categorias: nenhum transtorno mental } \\
\text { (nenhuma perturbação; perturbação leve) e transtorno } \\
\text { mental (depressão atípica; outras neuroses; transtorno de } \\
\text { personalidade; depressão maior; esquizofrenia residual; } \\
\text { outras psicoses funcionais; psicose orgânica). }\end{array}$ \\
\hline $\begin{array}{l}\text { Minimum Data Set } \\
\text { Depression Rating Scale } \\
\text { (MDSDRS) }^{* * *} \\
\text { Burrows et al. }^{21}\end{array}$ & $\begin{array}{l}\text { EUA e } \\
\text { Canadá }\end{array}$ & 3 & Depressão & $\begin{array}{l}\text { Composto por } 16 \text { indicadores de humor. As nove } \\
\text { expressões verbais são: declarações negativas, dúvidas } \\
\text { repetitivas, pedidos por ajuda repetitivos, raiva } \\
\text { persistente e irritabilidade, autodepreciação, medos } \\
\text { irreais, expressões de pânico, queixas repetidas de saúde } \\
\text { e queixas repetitivas de ansiedade. As sete expressões não } \\
\text { verbais são: variação de humor cotidiana, distúrbios do } \\
\text { sono, expressão facial de dor, tristeza e aflição, choro, } \\
\text { movimentos repetitivos (agitação), desistir de atividades } \\
\text { de interesse e interação social reduzida. }\end{array}$ \\
\hline
\end{tabular}


Quadro 3. continuação

\begin{tabular}{|c|c|c|c|c|}
\hline Nome e Referência & País & $\mathrm{N}$ cit & Variáveis & Descrição do instrumento \\
\hline $\begin{array}{l}\text { Geriatric Depression Scale } \\
\text { (GDS-12R) } \\
\text { Sutcliffe et al. } .^{23}\end{array}$ & Inglaterra & 2 & Depressão & $\begin{array}{l}\text { Baseada na versão do GDS-15. Foram removidos três } \\
\text { itens que não eram apropriados para ILPIs: 1) Você } \\
\text { prefere ficar em casa, ao invés de sair e fazer coisas novas?; } \\
\text { 2) Você sente que tem mais problemas com a memória do } \\
\text { que a maioria das pessoas?; 3) Você acha que a maioria } \\
\text { das pessoas são melhores do que você? }\end{array}$ \\
\hline $\begin{array}{l}\text { Research Mattis Dementia } \\
\text { Rating Scale (RMDRS) } \\
\text { Teresi, Kleinman e Ocepek- } \\
\text { Welikson }^{24}\end{array}$ & EUA & 1 & Cognição & $\begin{array}{l}\text { Foi realizada adaptação para capturar a gravidade das } \\
\text { deficiências existentes entre os idosos institucionalizados. } \\
\text { Contém os mesmos itens do original, no entanto, as } \\
\text { instruções para a administração são padronizadas e mais } \\
\text { estruturadas. Códigos são incluídos para distinguir entre } \\
\text { os dados desaparecidos devido à recusa, a incapacidade } \\
\text { física ou a diferentes níveis de não compreensão. }\end{array}$ \\
\hline $\begin{array}{l}\text { Algase Wandering Scale - } \\
\text { Version } 2 \text { (AWSV2) } \\
\text { Algase et al. } .^{29}\end{array}$ & $\begin{array}{c}\text { EUA e } \\
\text { Austrália }\end{array}$ & 1 & $\begin{array}{l}\text { Peregrinação } \\
\text { em idosos com } \\
\text { demência }\end{array}$ & $\begin{array}{l}\text { Mensura o comportamento de peregrinação (vagar) } \\
\text { característico na demência. É composta por } 38 \text { itens e foi } \\
\text { projetado para capturar elementos de frequência, padrão } \\
\text { ou qualidade, limite de transgressão, déficits de navegação } \\
\text { ou forma de averiguação e distribuição temporal. Utiliza } \\
\text { uma escala ordinal para cada item e que indicam de } \\
\text { ausência até alta frequência do comportamento. }\end{array}$ \\
\hline $\begin{array}{l}\text { Geriatric Depression Scale } \\
\text { (GDS 8) } \\
\text { Jongenelis et al. } .^{35}\end{array}$ & Holanda & 1 & Depressão & $\begin{array}{l}\text { Apresenta oito itens: você está basicamente satisfeito } \\
\text { com sua vida? Você sente que a sua vida está vazia? Você } \\
\text { costuma ficar entediado? Você está de bom humor a } \\
\text { maior parte do tempo? Você costuma se sentir impotente? } \\
\text { Você acha que é maravilhoso estar vivo? Você sente que } \\
\text { sua situação é desesperadora? }\end{array}$ \\
\hline $\begin{array}{l}\text { Brief Interview of Mental } \\
\text { Status (BIMS) } \\
\text { Chodosh et al. }{ }^{38}\end{array}$ & EUA & 1 & Cognição & $\begin{array}{l}\text { É uma entrevista de } 15 \text { itens que avalia três domínios: } \\
\text { memória, orientação e julgamento. A versão piloto } \\
\text { consistia em: recordação imediata (registro), recordação } \\
\text { tardia (podendo receber sugestões), itens de orientação } \\
\text { sobre dia, mês e ano corretos. }\end{array}$ \\
\hline $\begin{array}{l}\text { Cornell Scale for Depression } \\
\text { in Dementia, modified for } \\
\text { use by long-term care staff } \\
\text { (CSDD-M-LTCS) } \\
\text { Watson et al. }{ }^{44}\end{array}$ & EUA & 1 & Depressão & $\begin{array}{l}\text { Possui } 19 \text { itens que fazem uma avaliação da depressão, } \\
\text { do humor, do comportamento físico e dos sinais de } \\
\text { distúrbios ideacionais nos últimos sete dias. A fim } \\
\text { de tornar o instrumento acessível a não clínicos, o } \\
\text { formulário foi modificado para remover a linguagem } \\
\text { técnica e fornecer exemplos cotidianos. }\end{array}$ \\
\hline $\begin{array}{l}\text { Nijmegen Observer-Rated } \\
\text { Depression Scale (NORD) } \\
\text { Leontjevas et al. }{ }^{46}\end{array}$ & Holanda & 1 & Depressão & $\begin{array}{l}\text { Escala curta para rastreamento da depressão em idosos } \\
\text { com ou sem demência residentes em ILPIs. Os seis itens } \\
\text { são classificados como ausente e presente para sintomas } \\
\text { observáveis dos critérios de diagnóstico para transtorno } \\
\text { depressivo. }\end{array}$ \\
\hline \multicolumn{5}{|c|}{ Aspectos sociais } \\
\hline $\begin{array}{l}\text { Index for Social Engagement } \\
\text { (ISE) } \\
\text { Mor et al. }{ }^{* 6}\end{array}$ & EUA & 1 & $\begin{array}{l}\text { Engajamento } \\
\text { social }\end{array}$ & $\begin{array}{l}\text { Escala observacional constituída de seis questões } \\
\text { dicotômicas do MDS: vontade de interagir com os outros, } \\
\text { vontade de fazer planejamentos ou estruturar laços, } \\
\text { facilidade de iniciar atividades; estabelecer os próprios } \\
\text { objetivos, ter envolvimento na vida dentro da ILPI, } \\
\text { aceitar convites para a maioria das atividades. }\end{array}$ \\
\hline $\begin{array}{l}\text { Self-Report Relational Care } \\
\text { Scale (RCS) } \\
\text { McGilton et al. } .^{33}\end{array}$ & Canadá & 1 & $\begin{array}{l}\text { Cuidado } \\
\text { relacional }\end{array}$ & $\begin{array}{l}\text { A escala foi projetada especificamente para avaliar os } \\
\text { cuidados que a equipe de enfermagem proporciona } \\
\text { aos residentes que vivem em ILPIs. Possui seis itens } \\
\text { que incluem a qualidade da abordagem relacional dos } \\
\text { funcionários com o residente, além da confiança e da } \\
\text { satisfação do paciente. }\end{array}$ \\
\hline
\end{tabular}

Nota: ${ }^{* * *}$ Instrumentos derivados do Minimum Data Set/Resident Assessment Instrument (MDS/RAI). N cit= número de citações dos instrumentos nos 30 artigos identificados nesta revisão. AVDs= Atividades Básicas de Vida Diária/ILPIs= Instituições de Longa Permanência para Idosos. 
A presente revisão identificou uma heterogeneidade de instrumentos desenvolvidos para avaliação dos residentes em ILPIs, os quais englobam vários aspectos, sejam estes físicos, psicológicos, sociais ou multidimensionais.

Um dos principais resultados desta pesquisa evidencia que os Estados Unidos (EUA) foram o país que mais desenvolveu instrumentos para avaliação do idoso residente em ILPI. O alto investimento na pesquisa e a política nacional de atenção ao idoso contribuem para a implementação de constantes processos avaliativos com vistas à manutenção e ao incremento da qualidade do atendimento prestado aos idosos institucionalizados. Frente a esta dinâmica, o Congresso dos EUA criou uma legislação denominada OBRA 87, que estabelece um conjunto de padrões mínimos de atendimento e de direitos para as pessoas que vivem em instalações desta natureza. Esta regulamentação preconiza a utilização do Instrumento de Avaliação do Residente (RAI), de maneira padronizada, em todo o território nacional ${ }^{48,57,58}$.
O RAI é composto por duas partes interligadas, tais como: um conjunto mínimo de dados (MDS) e um conjunto amplo com uma série de avaliações dos Residentes (PAR). O MDS é um formulário com questões sobre a saúde, funcionamento e bem-estar dos moradores. O PAR é um conjunto de protocolos para uma análise mais aprofundada dos problemas que são frequentemente encontrados nas ILPIs ${ }^{57-59}$. O MDS foi o instrumento mais citado entre os 28 identificados nos 30 artigos selecionados nesta revisão. Constata-se que sete outros instrumentos (CPS, MDSDRS, MDS-ADL, MDS-COGS, ISE, MDSCHESS, MDS Section K) derivam do MDS. Evidencia-se, portanto, que esse instrumento permite um recorte em suas questões para a identificação de condições específicas conforme o interesse do profissional ${ }^{31,58,60}$.

No Brasil, a Política Nacional de Saúde da Pessoa Idosa relata que os idosos diferem entre si e que necessitam de uma avaliação pautada no conhecimento científico, condizente com as suas peculiaridades e adaptada à realidade sociocultural em que estão inseridos. Para tanto, incentiva a utilização de instrumentos técnicos validados, como o de avaliação funcional e psicossocial para detecção precoce de distúrbios relacionados às condições de saúde ${ }^{13}$. Complementarmente, a Resolução da Diretoria Colegiada 283 da Anvi$\mathrm{sa}^{12}$ estabelece a notificação anual e obrigatória de várias condições de saúde e a identificação do

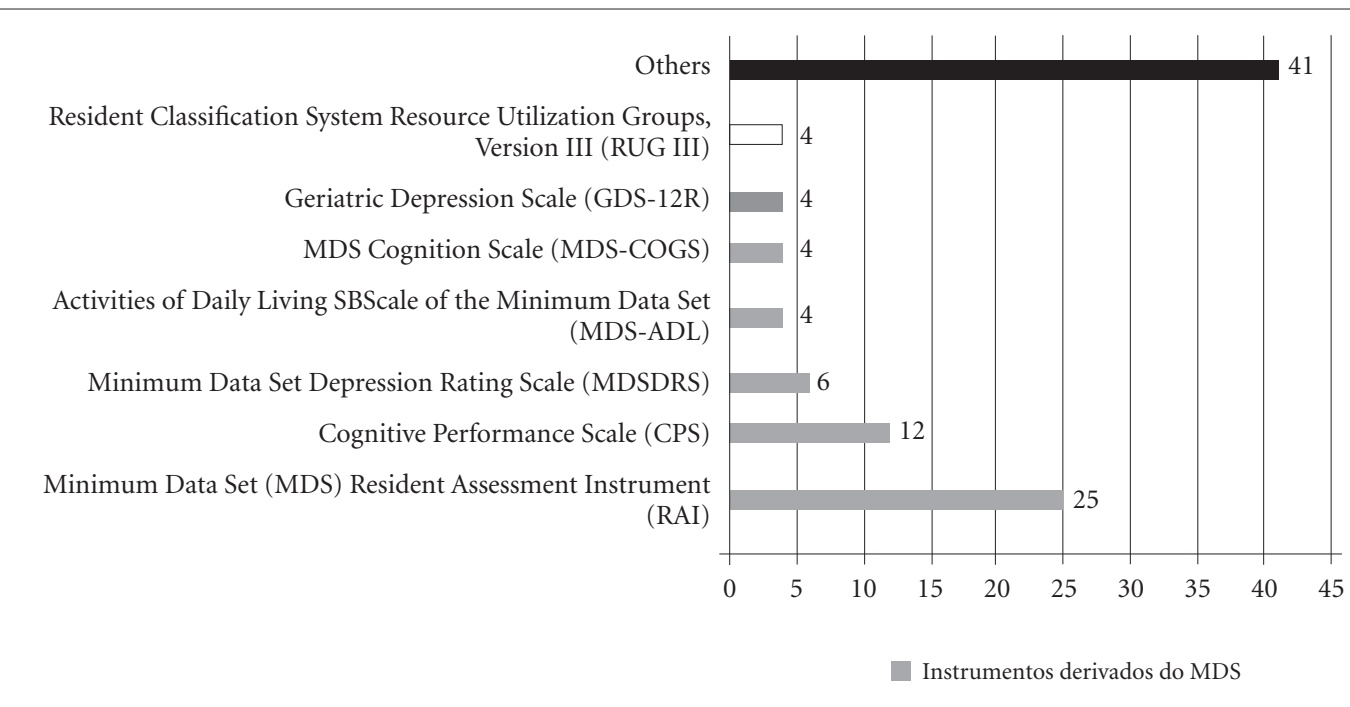

Figura 2. Porcentagem que os instrumentos foram citados em relação ao número total de citações. 
estado funcional dos idosos institucionalizados. Contudo, na prática, a avaliação sistemática dos idosos residentes em ILPIs tem se mostrado de difícil implementação, o que condiz com o fato desta revisão não ter encontrado nenhum instrumento desenvolvido em território nacional.

Apesar da existência de algumas propos$\operatorname{tas}^{7,61,62}$ para a sistematização da avaliação dos idosos institucionalizados, não existe no Brasil um protocolo único que oriente o processo de avaliação e crie um banco de dados nacional com informações referentes a essa população. Sendo assim, torna-se urgente que esforços sejam realizados nesse sentido, para fazer cumprir o que determina a legislação brasileira, favorecendo a obtenção de elementos que possam ser úteis tanto clinica quanto socialmente.

Benedetti et al. ${ }^{63}$, ao aplicar a bateria de aptidão física denominada AAHPERD em idosos institucionalizados, observaram que estes apresentavam dificuldades na execução de alguns testes, devido a suas limitações físico-funcionais, havendo a necessidade de realizar todo o processo de adaptação das baterias às condições desses idosos.

Outra conjuntura ocorre quando o instrumento é criado especificamente para a população idosa institucionalizada, como é o caso do Oral Health Assessment Tool, cuja função é fornecer aos cuidadores de idosos em ILPIs um instrumento simples para avaliar a saúde bucal dos residentes $^{64}$. Este instrumento sofreu um processo de validação para a realidade brasileira ${ }^{65}$. Com isso, entende-se que a aplicação de instrumentos de medida é algo complexo, que requer seriedade e rigor científico no sentido de garantir a validade e a fidedignidade. Assim, cabe uma reflexão sobre o processo de avaliação dos residentes em ILPIs, englobando a adequação dos instrumentos de medida e a capacitação técnica dos distintos avaliadores.

A depressão, a cognição e a capacidade funcional, foram, respectivamente, as variáveis mais identificadas nos estudos em questão. A alta prevalência de depressão no contexto institucional ${ }^{66-68}$ está frequentemente relacionada a fatores como limitação e/ou dependência e insatisfação com a instituição ${ }^{69}$. Para esta variável, os itens buscam principalmente identificar a presença de sinais e sintomas que remetam a problemas de comportamento, sentimentos negativos e ausência de bem-estar, através de comunicação verbal e não verbal, o que vai ao encontro dos achados de pesquisadores prévios ${ }^{70}$.

O declínio cognitivo tem alta prevalência entre os idosos residentes nessas instituições ${ }^{71,72}$.
A prevenção e a detecção precoce de alterações cognitivas, assim como a adoção de tratamentos a curto e longo prazo, com vistas à manutenção da qualidade de vida, ainda são os maiores desafios já documentados ${ }^{73}$. Nos instrumentos analisados $^{18,24,38,55}$, os itens avaliam principalmente questões referentes a dificuldades de raciocínio, desorientação tempo/espacial no ambiente da instituição, comunicação prejudicada e tomada de decisão para avaliar a capacidade cognitiva dos residentes.

Ainda, o alto índice de decréscimo funcional na população idosa institucionalizada foi documentado por vários estudos ${ }^{74-76}$. Gonçalves et al. ${ }^{77}$ consideram importante avaliar a capacidade funcional com o propósito de discriminar o déficit nos diferentes componentes da aptidão física, classificando devidamente os residentes. Assim, para a capacidade funcional, os instrumentos de caráter multidimensional ${ }^{41,47-50}$ utilizam critérios baseados nas condições e no funcionamento físico dos idosos, além de avaliar a necessidade e a dependência de cuidados requeridos pelo residente. Outros instrumentos encontrados nesta revisão ${ }^{43,52,53}$ avaliam a capacidade funcional através do desempenho em tarefas dentro da rotina da ILPI, destacando-se a capacidade de se vestir, transferências e locomoção.

Os instrumentos MDS/RAI ${ }^{48}$ e MALFA ${ }^{49}$ possuem caráter multidimensional, possibilitando uma visão geral sobre o perfil de saúde, bem-estar e funcionamento do idoso dentro da instituição. Estes instrumentos auxiliam nas diagnoses e permitem um planejamento das intervenções. Os itens do MDS permitem, ainda, a análise da tomada de decisões, as expressões verbais e não verbais, o desempenho nas atividades básicas de vida diária (AVDs), a interação com outros residentes e o envolvimento em distintas atividades dentro da ILPI.

Os instrumentos GDS $12-\mathrm{R}^{23}$, GDS $-8^{35} \mathrm{e}$ $\mathrm{NORD}^{46}$ buscam identificar sintomas depressivos. Por outro lado, Siqueira et al. ${ }^{78}$, ao identificarem a alta prevalência de depressão entre os idosos, ressaltam a importância da criação de uma escala mais simples e de fácil compreensão dirigida ao público com baixo nível cognitivo. Tal fato sugere o desenvolvimento de novos estudos com o objetivo de comparar a depressão nas populações de idosos com graus variados de capacidade cognitiva.

Belgian ADL-Evaluation Scale ${ }^{52}, \mathrm{CCAM}^{43} \mathrm{e}$ $\mathrm{SIT}^{30}$ são instrumentos que avaliam tarefas funcionais e motoras, associados à orientação temporo-espacial na ILPI. Dutch Care Dependence 
Scale-CDS ${ }^{50}$, Time in Care needs - TIC- $\mathrm{n}^{41}$ e Self-Report Relational Care Scale-RCS ${ }^{33}$ buscam avaliar a dependência que essa população possui em relação aos cuidados e à necessidade de apoio em face à fragilidade e ao comprometimento funcional e cognitivo.

Outros instrumentos úteis e interessantes para esse contexto são o Algase Wandering Scale - Version 2 (AWSV2) ${ }^{29}$, que avalia o comportamento errante principalmente associado à Doença de Alzheimer, e o Resource Utilization Groups, Version III (RUG-III), que foi construído para avaliar os custos de cada residente na instituição. Os gastos que cada residente demanda são elevados e essa ferramenta auxiliaria no planejamento dos serviços prestados, caso seja incorporada à rotina destas instituições ${ }^{47}$. Dessa maneira, seria um instrumento importante para auxiliar os gestores das ILPIs filantrópicas e públicas, que normalmente encontram dificuldades financeiras para a manutenção de suas atividades.

Em síntese, existem várias opções de instrumentos a serem utilizados em avaliações ou pesquisas no ambiente institucional, e sua seleção vai depender das variáveis de interesse (físicas, psicológicas ou sociais) por parte dos pesquisadores. No caso de avaliações padronizadas no período da admissão ou na rotina de trabalho dos profissionais da equipe de saúde, acredita-se que o instrumento Minimum Data Set/Resident Assessment Instrument (MDS/RAI), por seu caráter multidimensional e por já ser bastante utilizado em outros países, seja um dos mais adequados. No entanto, todos os instrumentos apresentados devem passar por um processo de validação/adaptação transcultural para a realidade brasileira. Além disso, a maioria dos instrumentos baseia-se na observação direta do residente, o que facilita a avaliação dos indivíduos sem a capacidade cognitiva preservada, algo comum nesse contexto.

Logo, seguindo os pressupostos de Hedrick ${ }^{79}$, entende-se que os instrumentos de avaliação devam ser utilizados com ponderações pelo pesquisador, não devendo substituir as avaliações não padronizadas como a anamnese e o exame físico. Paixão e Reichenheim ${ }^{11}$ consideram que as medidas padronizadas possuem a vantagem de serem menos suscetíveis a vieses, mais acuradas e reprodutíveis, mesmo quando utilizadas por equipes com diferentes níveis de treinamento. $\mathrm{O}$ uso de testes padronizados permite ao profissional de saúde estabelecer uma linha de base bem definida sobre futuras decisões clínicas.

As especificidades do público-alvo das avaliações devem ser consideradas, visto que isso pode facilitar, ou até mesmo inviabilizar, a aplicação de alguns instrumentos. Silva e Santos ${ }^{80}$ relatam ser visível o despreparo dos trabalhadores que assistem os idosos institucionalizados. Portanto, o investimento na pesquisa e a capacitação de recursos humanos ainda parece ser a melhor conduta.

\section{Considerações finais}

A partir dos resultados, foi possível concluir que os EUA são o país que mais elabora instrumentos voltados a essa população. A existência de uma legislação que obriga as ILPIs a realizarem um protocolo de avaliação padronizado, formando um cadastro único, pode explicar em parte tal desempenho. $\mathrm{O}$ instrumento multidimensional denominado Minimum Data Set/Resident Assessment Instrument (MDS/RAI) foi o mais utilizado nos estudos da presente revisão. As variáveis mais frequentemente avaliadas pelos instrumentos foram a depressão, a cognição e a capacidade funcional, as quais estão associadas com as características dos residentes em instituições de longa permanência. A maioria dos instrumentos baseia-se na observação direta do residente, o que facilita a aplicabilidade do instrumento no contexto institucional, pois não necessita da capacidade cognitiva preservada deste para sua utilização.

O Brasil não aparece nesta revisão, o que evidencia a carência de estudos e estimula a criação e a reformulação de políticas públicas que garantam um sistema de avaliação multidimensional padronizado aos residentes de ILPIs no país. Através de um processo sistemático e contínuo de coleta, análise, interpretação e disseminação de informações pode ser possível ampliar a vigilância epidemiológica dessa população em todo território nacional.

O presente estudo possui algumas limitações, pois poderia ter utilizado um número maior de bases de dados, assim como outros idiomas. Ainda, durante o processo de análise dos artigos selecionados encontrou-se dificuldades de acesso aos instrumentos na íntegra, o que impossibilitou a obtenção de maiores informações sobre suas características.

Diante destes resultados, sugerem-se futuros estudos voltados à criação e/ou à adaptação transcultural de instrumentos que respeitem o arcabouço conceitual existente e contemplem as características peculiares dessa população, segundo os princípios psicométricos. Além disso, torna-se um desafio que as descobertas prove- 
nientes das pesquisas sobre instrumentos de avaliação consigam se efetivar na prática cotidiana dos profissionais das ILPIs, através da sua apro- priação e utilização como uma estratégia para melhorar o gerenciamento e o cuidado desses idosos.

\section{Colaboradores}

PA Medeiros participou de todas as etapas de elaboração do artigo; AR Fortunato e AAF Viscardi participaram da pesquisa, análise e interpretação dos dados; FF Sperandio participou revisão crítica e aprovação da versão final e GZ Mazo orientou todas as etapas de construção do trabalho.

\section{Agradecimentos}

Aos Professores Fernando Luiz Cardoso e Tânia Rosane Bertoldo Benedetti pelo auxílio em algumas etapas deste estudo. Ao Conselho Nacional de Desenvolvimento Científico e Tecnológico $\mathrm{CNPq}$ pela bolsa de produtividade em pesquisa destinada à orientadora deste estudo e à Coordenação de Aperfeiçoamento de Pessoal de Nível Superior - CAPES pela bolsa de mestrado concedida ao autor Paulo Adão de Medeiros.

\section{Referências}

1. Creutzberg M, Goncalves LHT, Sobottka EA, Ojeda BS. A Instituição de Longa Permanência Para Idosos e o Sistema de Saúde. Rev Latino-Am Enfermagem 2007; 15(6):1144-1149.

2. Camarano AA. Cuidados de longa duração para a população idosa: família ou instituição de longa permanência? Sinais Sociais 2008; 7(3):10-39.

3. Camargos MCS. Instituições de longa permanência para idosos: um estudo sobre a necessidade de vagas. Rev. Bras. Estud. Popul 2014; 31(1):211-217.

4. Piexak DR, Freitas PH, Backes DS, Moreschi C, Ferreira CLL, Souza MHT. Percepção de profissionais de saúde em relação ao cuidado a pessoas idosas institucionalizadas. Rev. Bras. Geriatr. Gerontol 2012; 15(2):201-208.

5. Santos SR. Cultura nas instituições de saúde e suas relações com a identidade individual. Cogitare Enferm 2007; 12(2):229-235.

6. Felician AM, Santos SSC, Pelzer MT, Oliveira AM, Pinho LB. Construção de ferramenta avaliativa direcionada às instituições de longa permanência para idosos: relato de experiência. Rev. Eletr. Enf 2011; 13(3):474-482.

7. Ribeiro RC, Marin HF. A proposal for a health evaluation tool for the institucionalized aged based on the concept of nursing minimum data set. Rev Bras Enferm 2009; 62(2):204-212.

8. Coluci MZO, Alexandre NMC, Milani D. Construção de instrumentos de medida na área da saúde. Cien Saude Colet 2015; 20(3):925-936.

9. DeSousa DA, Moreno AL, Gauer G, Manfro GG, Koller SH. Revisão sistemática de instrumentos para avaliação de ansiedade na população brasileira. Aval. Psicol 2013; 12(3):397-410.

10. Alexandre NMC, Coluci MZO. Validade de conteúdo nos processos de construção e adaptação de instrumentos de medidas. Cien Saude Colet 2011; 16(7):3061-3068.

11. Paixão Júnior CM, Reichenheim ME. Uma revisão sobre instrumentos de avaliação do estado funcional do idoso. Cad Saude Publica 2005; 21(1):7-19.

12. Brasil. Resolução da Diretoria Colegiada - RDC no 283, de 26 de setembro de 2005. Dispõe sobre Regulamento Técnico que define as normas de funcionamento para as instituições de longa permanência para idosos. Agência Nacional de Vigilância Sanitária 2005; 26 set.

13. Brasil. Ministério da Saúde (MS). Portaria nº 2.528 de 19 de outubro de 2006. Aprova a Política Nacional de Saúde da Pessoa Idosa. Diário Oficial da união 2006; 19 out.

14. Prisma - Preferred Reporting Items for Systematic Reviews and Meta-Analyses [internet]. 2009. [acessado 2013 maio 20]. Disponível em: http://www.prisma -statement.org/

15. Liberati A, Altman DG, Tetzlaff J, Mulrow C, Gøtzsche PC, Ioannidis JPA, Clarke M, Devereaux PJ, Kleijnen J, Moher D. The PRISMA statement for reporting systematic reviews and meta-analyses of studies that evaluate health care interventions: explanation and elaboration. Ann Intern Med 2009; 151(4):W65-W94. 
16. Sign - Scottish Intercollegiate Guidelines Network. [internet]. 2001. [acessado 2013 maio 25]. Disponível em: http://www.sign.ac.uk/methodology/filters.html

17. Frisoni GB, Franzoni S, Rozzini R, Ferrucci L, Boffelli $\mathrm{S}$, Trabucchi M. A nutritional index predicting mortality in the nursing home. J Am Geriatr Soc 1994; 42(11):1167-1172.

18. Hartmaier SL, Sloane PD, Guess HA, Koch GG. The MDS Cognition Scale: a valid instrument for identifying and staging nursing home residents with dementia using the minimum data set. J Am Geriatr Soc 1994; 42(11):1173-1179.

19. Isohanni M, Winblad I, Nieminen P, Hiltunen P, Spalding M. A Short DSM-111-R-Based Diagnostic Instrument for Screening Mental Disorders in Geriatric Institutions. Int Psychogeriatr 1996; 8(3):459-468.

20. Björkgren MA, Finne-Soveri H, Fries BE. Validity and Reliability of Resource Utilization Groups (RUG III) in Finnish Long-Term Care Facilities. Scand J Publ Health 1999; 27(3):228-234.

21. Burrows AB, Morris JN, Simon SE, Hirdes JP, Phillips $\mathrm{CH}$. Development of a minimum data set-based depression rating scale for use in nursing homes. Age Ageing 2000; 29(2):165-172.

22. Gruber-Baldini AL, Zimmerman SI, Mortimore E, Magaziner J. The validity of the Minimum Data Set in measuring the cognitive impairment of persons admitted to nursing homes. J Am Geriatr Soc 2000; 48(12):1601-1606.

23. Sutcliffe C, Cordingley L, Burns A, Mozley CG, Bagley $\mathrm{H}$, Huxley P, Challis D. A new version of the Geriatric depression Scale for nursing and residential home populations: The Geriatric Depression Scale (Residential) (GDS-12R). Int Psychogeriatr 2000; 12(2):173-181.

24. Teresi JA, Kleinman M, Ocepek-Wlikson K. Modern psycometric for detection of diff e rential item functioning: aplication to cognitive assessment measures. Statist Med 2000; 19(11-12):1651-1683.

25. Dijkstra A, Tiesinga LJ, Goossen WT, Dassen TW. Further psychometric testing of the Dutch Care Dependency Scale on two different patient groups. Int $J$ Nurs Pract 2002; 8(6):305-314.

26. McCurren C. Assessment for Depression Among Nursing Home Elders: Evaluation of the MDS Mood Assessment. Geriatric Nursing 2002; 23(2):103-108.

27. Anderson RL, Buckwalter KC, Buchanan RJ, Maas ML, Imhof SL. Validity and reliability of the Minimum Data Set Depression Rating Scale (MDSDRS) for older adults in nursing homes. Age Ageing 2003; 32(4):435-438

28. Oborne CA, Hooper R, Swift CG, Jackson SH. Explicit, evidence-based criteria to assess the quality of prescribing to elderly nursing home residents. Age Ageing 2003; 32(1):102-8.

29. Algase DL, Beattie ERA, Song JA, Milke D, Duffield C, Cowan B. Validation of the Algase Wandering Scale (Version 2) in a cross cultural sample. Aging Ment Health 2004; 8(2):133-142.

30. Miller WC, Miller F, Trenholm K, Grant D, Goodman K. Development and preliminary assessment of the measurement properties of the Seating Identification Tool (SIT). Clin Rehabil 2004; 18(3):317-325.

31. Stevenson KB, Moore JW, Sleeper B. Validity of the Minimum Data Set in Identifying Urinary Tract Infections in Residents of Long-Term Care Facilities. J Am Geriatr Soc 2004; 52(5):707-711.

32. Bowman JJ, Keller HH. Assessing Nutritional Risk of Long-Term Care Residents. Can J Diet Pract Res 2005; 66(3):155-161.
33. McGilton KS, Pringle DM, O’Brien-Pallas LL, Wynn F, Streiner D. Development and Psychometric Testing of the Relational Care Scale. J Nurs Manag 2005; 13(1):51-64.

34. Resnick B, Jung DY. Utility of the Maryland Assisted Living Functional Assessment Tool. J Hous Elderly 2006; 20(3):109-121.

35. Jongenelis K, Gerritsen DL, Pot AM, Beekman ATF, Eisses AMH, Kluiter H, Ribbe MW. Construction and validation of a patient- and user-friendly nursing home version of the Geriatric Depression Scale. Int J Geriatr Psychiatry 2007, 22(9):837-842.

36. Paquay L, Lepeleire J, Schoenmakers B, Ylieff M, Fontaine O, Buntinx F. Comparison of the diagnostic accuracy of the Cognitive Performance Scale (Minimum Data Set) and the Mini-Mental State Exam for the detection of cognitive impairment in nursing home residents. Int J Geriatr Psychiatry 2007; 22(4):286-293.

37. Auret KA, Toye C, Goucke R, Kristjanson LJ, Bruce D, Schug S. Development and testing of a modified version of the brief pain inventory for use in residential aged care facilities. J Am Geriatr Soc 2008; 56(2):301-306.

38. Chodosh J, Edelen MO, Buchanan JL, Yosef JA, Ouslander JG, Berlowitz DR, Streim JE, Saliba D. Nursing home assessment of cognitive impairment: development and testing of a brief instrument of mental status. J Am Geriatr Soc 2008; 56(11):2069-2075.

39. Delbaere K, Close JC, Menz HB, Cumming RG, Cameron ID, Sambrook PN, March LM, Lord SR. Development and validation of fall risk screening tools for use in residential aged care facilities. Med J Aust 2008; 189(4):193-196.

40. Gerritsen DL, Steverink N, Frijters DH, Hirdes JP, Ooms ME, Ribbe MW. A revised Index for Social Engagement for long-term care. J Gerontol Nurs 2008; 34(4):40-48.

41. Thorsell KB, Nordström B, Nyberg P, Sivberg BV. Measuring care of the elderly: psychometric testing and modification of the Time in Care instrument for measurement of care needs in nursing homes. BMC Geriatrics 2008; 8(22):2-8.

42. Worden A, Challis D, Hancock G, Woods R, Orrell M. Identifying need in care homes for people with dementia: the relationship between two standard assessment tools. Aging Ment Health 2008; 12(6):719-728.

43. Huijbregts MP, Teare GF, McCullough C, Kay TM, Streiner D, Wong SK, McEwen SE, Otten I. Standardization of the continuing care activity measure: a multicenter study to assess reliability, validity, and ability to measure change. Phys Ther 2009; 89(6):546-555.

44. Watson LC, Zimmerman S, Cohen LW, Dominik R. Practical Depression Screening in Residential Care/ Assisted Living: Five Methods Compared to Gold Standard Diagnoses. Am J Geriatr Psychiatry 2009; 17(7):556-564

45. Berlowitz DR, Hickey EC, Saliba D. Can administrative data identify active diagnoses for long-term care resident assessment? J Rehabil Res Dev 2010; 47(8):719-724.

46. Leontjevas R, Gerritsen DL, Vernooij-Dassen MJFJ, Teerenstra S, Smalbrugge M, Koopmans RTCM. Nijmegen Observer-Rated Depression scale for detection of depression in nursing home residentes. Int J Geriatr Psychiatry 2012; 27(10):1036-1044.

47. Fries BE, Cooney L. Resource utilization groups: a patient classification system for long term care. Med Care 1985; 23(2):110-122. 
48. Morris JN, Hawes C, Fries BE, Phillips CD, Mor V, Katz S, Murphy K, Drugovich ML, Friedlob AS. Designing the National Resident Assessment Instrument for Nursing Homes. Gerontologist 1990; 30(3):293-307.

49. Resnick B, Jung DK. Using the Maryland Assisted Living Functional Assessment to Establish a Plan of Care. Assisted Living Consult 2005; 1(4):20-27.

50. Dijkstra A, Buist G, Dassen TWN. Operationalization of the concept of 'Nursing Care Dependency' for use in long term care facilities. Aust N Z J Ment Health Nurs 1998; 7(4):142-151.

51. Canadian Institute for Health Information (CIHI). Minimum Data Set v 2.0 user's manual. Toronto: CIHI; 1996.

52. Arnaert A, Delesie L. Calibration of measurement data: the Belgian Institute of Health Insurance Index of ADL. [IJking van meetgegevens: RIZIV A.D.L.-index]. Acta Hospitalia 1999; 39:9-31.

53. Morris JN, Pries BE, Morris AS. Scaling ADLs Within the MDS. Journal of Gerontology: Medical Sciences 1999; 54(II):M546-M553.

54. Hirdes JP, Frijters DH, Teare GF. The MDS-CHESS scale: a new measure to predict mortality in institutionalized older people. J Am Geriatr Soc 2003; 51(1):96-100.

55. Morris JN, Fries BE, Mehr DR, Hawes C, Phillips C, Mor V, Lipsitz LA. MDS Cognitive Performance Scale. J Gerontol 1994; 49(4):M174-182.

56. Mor V, Branco K, Fleishman J, Hawes C, Phillips C, Morris J, Fries B. The structure of social engagement among nursing home residents. J Gerontol B Psychol Sci Soc Sci 1995; 50(1):P1-P8

57. Hawes C, V Mor, CD Phillips, Fries BE, Morris JN, Steele-Friedlob E, Greene AM, Nennstiel M. The OBRA-87 nursing home regulations and implementation of the Resident Assessment Instrument: effects on process quality. J Am Geriatr Soc 1997; 45(8):977-985.

58. Achterberg WP. The use of the Minimum Data Set (MDS) for research into quality of care and patient functioning in nursing homes [thesis]. Amsterdam: Institute for Research in Extramural Medicine (EMGO Institute); 2004.

59. Morris JN, Hawes C, Murphy K, Nonemaker S. Resident assessment instrument: training manual and resource guide. Natick: Elliot Press; 1991.

60. Mor VA. Comprehensive clinical assessment tool to inform policy and practice:applications of the minimum data set. Med Care 2004; 42 (4 Supl.):50-59.

61. Petri AC, Bastos LC. Sistema Informatizado de Controle de Dados do Idoso Institucionalizado. In: Anais do X Congresso Brasileiro de Informática em Saúde; 2006; Florianópolis. [acessado 2015 abr 30]. Disponível em: http://www.sbis.org.br/cbis/arquivos/738.pdf.

62. Prefeitura Municipal de Belo Horizonte (PMBH). Protocolo de avaliação admissional multidimensional do idoso institucionalizado. Belo Horizonte: Secretaria Municipal de Saúde [internet]. 2004. [acessado 2015 abr 10]. Disponível em: http://www.pbh.gov.br/smsa/ biblioteca/geas/protocolodeatencaoasaudedoidoso.doc.

63. Benedetti TRB, Mazo GZ, Gonçalves LHT. Bateria de testes da AAHPERD: adaptação para idosos institucionalizados. Rev Bras Cineantropom Desempenho Hum 2014; 16(1):1-14.

64. Chalmers JM, King PL, Spencer AJ, Wright FA, Carter $\mathrm{KD}$. The oral health assessment tool: validity and reliability. Aust Dent J 2005; 50(3):191-199.
65. Goncalves LHT, Mello ALSF, Zimermann K. Validação de instrumento de avaliação das condições de saúde bucal de idosos institucionalizados. Esc. Anna Nery 2010; 14(4):839-847.

66. Heiser D. Depression identification in the long-term care setting: the GDS vs. the MDS. Rev Clin Gerontol 2004; 27(4):3-18.

67. Thakur M, Blazer DG. Depression in long-term care. J Am Med Dir Assoc 2008; 9(2):82-87.

68. Vaz SFA, Gaspar NMS. Depressão em idosos institucionalizados no distrito de Bragança. Rev. Enf. Ref 2011; III(4):27-32.

69. Silva ER, Sousa ARP, Ferreira LB, Peixoto HM. Prevalência e fatores associados à depressão entre idosos institucionalizados: subsídio ao cuidado de enfermagem. Rev. Esc. Enferm. USP 2012; 46(6):1387-1393.

70. Andrade ACA, Lima FRA, Silva LFA, Santos SSC. Depressão em idosos de uma Instituição de Longa Permanência: proposta de ações de enfermagem. Rev Gaúcha Enferm 2005; 26(1):57-66.

71. Ferretti CEL. Identificação de fatores de risco envolvidos no processo de institucionalizacäo do portador de demência [tese]. São Paulo: Universidade Federal de São Paulo; 2004.

72. Scocco P, Rapattoni M, Fantoni G. Nursing home institutionalization: a source of eustress or distress for the elderly? Int J Geriatr Psychiatry 2006; 21(3):281-287.

73. Reis LA, Torres GV, Araújo CC, Reis LA, Novaes LKN. Rastreamento cognitivo de idosos institucionalizados no município de Jequié-BA. Psicol. Estud 2009; 14(2):295-301.

74. Guedes JM, Silveira RCR. Análise da capacidade funcional da população geriátrica institucionalizada da cidade de Passo Fundo - RS. RBCEH 2004; 1(2):10-21.

75. Ferrantin AC, Borges, CF, Morelli JGS, Rebelatto JR. A execução de AVDs e mobilidade functional em idosos institucionalizados e não institucionalizados. Fisioter. mov 2007; 20(3):115-121.

76. Aires M, Paz AA, Perosa CT. Situação de saúde e grau de dependência de pessoas idosas institucionalizadas. Rev Gaúcha Enferm 2009; 9(30):492-499.

77. Gonçalves LHT, Silva AH, Mazo GZ, Benedetti TRB, Santos SMA, Marques S, Rodrigues RAP, Portella MR, Scortegagna HM, Santos SSC, Pelzer MT, Souza AS, Meira EC, Sena ELS, Creutzberg M, Rezende TL. O idoso institucionalizado: avaliação da capacidade funcional e aptidão física. Cad Saude Publica 2010; 26(9):1738-1746.

78. Siqueira GR, Vasconcelos DT, Duarte GC, Arruda IC, Costa JAS, Cardoso RO. Análise da sintomatologia depressiva nos moradores do Abrigo Cristo Redentor através da aplicação da Escala de Depressão Geriátrica (EDG). Cien Saude Colet 2009; 14(1):253-259.

79. Hedrick SC. Assessment of functional status: activities of daily living. In: Rubenstein LZ, Wieland D, Bernabei $\mathrm{R}$, editors. Geriatric assessment technology state of the art. Milano: Editrice Kurtis; 1995. p. 311.

80. Silva BT, Santos SSC. Cuidados aos idosos institucionalizados - opiniões do sujeito coletivo enfermeiro para 2026. Acta Paul Enferm 2010; 23(6):775-781.

Artigo apresentado em 06/06/2015

Aprovado em 15/09/2015

Versão final apresentada em 17/09/2015 